\title{
Conveying Intentions Through Haptics in Human-Computer Collaboration
}

\author{
Ayse Kucukyilmaz* $^{*}$ T. Metin Sezgin ${ }^{\dagger} \quad$ Cagatay Basdogan ${ }^{\ddagger}$ \\ College of Engineering \\ Koç University
}

\begin{abstract}
Haptics has been used as a natural way for humans to communicate with computers in collaborative virtual environments. Humancomputer collaboration is typically achieved by sharing control of the task between a human and a computer operator. An important research challenge in the field addresses the need to realize intention recognition and response, which involves a decision making process between the partners. In an earlier study [11], we implemented a dynamic role exchange mechanism, which realizes decision making by means of trading the parties' control levels on the task. This mechanism proved to show promise of a more intuitive and comfortable communication. Here, we extend our earlier work to further investigate the utility of a role exchange mechanism in dynamic collaboration tasks. An experiment with 30 participants was conducted to compare the utility of a role exchange mechanism with that of a shared control scheme where the human and the computer share control equally at all times. A no guidance condition is considered as a base case to present the benefits of these two guidance schemes more clearly. Our experiment show that the role exchange scheme maximizes the efficiency of the user, which is the ratio of the work done by the user within the task to the energy spent by her. Furthermore, we explored the added benefits of explicitly displaying the control state by embedding visual and vibrotactile sensory cues on top of the role exchange scheme. We observed that such cues decrease performance slightly, probably because they introduce an extra cognitive load, yet they improve the users' sense of collaboration and interaction with the computer. These cues also create a stronger sense of trust for the user towards her partner's control over the task.
\end{abstract}

\section{INTRODUCTION}

It is widely accepted that computers are better than humans in tasks requiring precision and accuracy [1]. On the other hand, humans have certain traits that make them suitable for tasks that require decision making and reasoning. However, many tasks require both. For example, CAD, surgical training, and entertainment applications can benefit from a human's intelligence, and a computer's precision if both are available simultaneously. For instance, in a CAD model assembly task, a human operator can quickly analyze the task, determine the assembly sequence, and roughly align the parts. Then, the computer can take control of the task and perform the fine adjustment of the parts. Such control trades constitute a role exchange process. Roles describe the authorities, the responsibilities, and the functions of the collaborating agents. In previous work, we have shown that a role exchange mechanism can be implemented in human-computer collaboration to allow each party to take leadership of the task whenever necessary [11]. We observed that the role exchange mechanism does not improve task perfor-

\footnotetext{
*e-mail: akucukyilmaz@ku.edu.tr

†e-mail:mtsezgin@ku.edu.tr

‡e-mail:cbasdogan@ku.edu.tr
}

IEEE World Haptics Conference 2011

21-24 June, Istanbul, Turkey

978-1-4577-0297-6/11/\$26.00 @2011 IEEE mance as much as an equal control guidance scheme does, however it is decreases the energy spent by the user and provides a subjectively pleasing interaction. However, in that study, the users were not informed on the existence of a role exchange mechanism, hence they failed to utilize it effectively in favor of the task. In this study, we extend the work in [11] to discover salient benefits of a role exchange mechanism when the users are explicitly instructed to use the mechanism. Additionally, we observed that when users face difficulties in understanding the control states within the task, they fail to utilize the role exchange mechanism effectively. Hence, we suggest that by emphasizing the control levels through certain sensory cues, we can achieve a better collaborative scheme.

Also, as collaborative tasks get complex and dynamic, beside exchanging roles, the parties need to employ mechanisms to adapt to each other [2]. This adaptation might require a process where parties discuss and negotiate to take certain roles. In this regard, the negotiation process requires the communication and interpretation of sensory cues. Ultimately, a system in which the computers can infer the human's intentions may prove to be effective in collaboration. In this study, we allowed the users to communicate their intentions on changing control levels through the haptic link. In return, we programmed the computer to react and adapt to the actions of the user.

This paper summarizes our attempts to reveal the benefits of defining and negotiating roles in haptic human-computer collaboration. Typically, roles allow the human and the computer to partition the task into units to get maximum benefit from each other's abilities. In our system, the human acts as the decision maker in setting the control level during a collaborative task. The human communicates her intentions through the haptic channel by varying the applied forces. Ultimately, we aim to enable a subjectively pleasing communication while maximizing task performance and user efficiency.

An experiment with 30 subjects has been conducted to measure the utility of the role exchange mechanism. In order to quantify the efficiency of the user, we define a metric that combines the energy spent by the user and the work done by her towards completing the task. Additionally, we seek the effectiveness of equipping the system with additional visual and vibrotactile informative cues that transfer the state of the interaction to the user. The main contributions of this study are listed below. We show that:

- the proposed role exchange mechanism improves the task performance,

- the user wastes less energy when the task involves a role exchange mechanism,

- the additional sensory cues increases the user's awareness of the control levels of the collaborating parties, improve the perceived level of collaboration and interaction of the user, and reinforce the users' belief that the computer will lead the task correctly.

This paper is organized as follows: In the next section, we present the related work. Section 3 presents the virtual environment used in the experiments as well as the haptic negotiation model, as 
introduced in [11], for dynamic and natural negotiation of roles, Section 4 lists the conditions used in the experiment and summarizes the experimental design along with the measures used in analysis. The results are discussed in Section 5, and finally Section 6 presents conclusions and future directions.

\section{Related Work}

In collaborative tasks involving haptics, force and vibrotactile cues have been used by partners to communicate with each other. Basdogan et al. [1] showed that haptics contributes significantly to the sensory communication in collaborative task. Haptics is often used for guidance in training applications to assist sensorimotor tasks, such as steering, calligraphy, and surgical training [4, 9, 4, 8, 12] and in real-time collaborative virtual environments [10]. Recently, it has been illustrated that progressive and predictive mechanisms, which alter the amount of guidance during the task, improve the learning rate of the users [6,5]. Alternatively, Lee and Choi [7] suggested that haptic disturbance can also be used to teach the dynamics of the task in the long run. In this study, we aim to provide haptic guidance to improve task performance. However, unlike the cited studies, we aim to develop a mechanism in which the human can display her intentions to the computer dynamically. Additionally, we aim to improve certain subjective qualities of interaction, such as comfort, the sense of collaboration, the level of interaction, the user's perception of the underlying guidance mechanism, and her belief towards computer guidance.

In [11], we suggested that haptic negotiation through a role exchange mechanism can further contribute to the collaborative experience. The definition of roles in haptic collaboration is an emerging topic. Reed and Peshkin [13] suggested specialization behaviors such as accelerator and decelerator roles in a target acquisition task. These roles were extracted regarding the interaction of two human operators. However, when the force profile acquired during human-human collaboration is replicated in the interaction of a human user and a computer, they failed to observe a similar specialization strategy. Stefanov et al. [14] defined conductor and executor roles for the partners and introduced a model to classify the roles based on the velocities and the interaction forces applied through the haptic devices controlled by two human users. Their interaction model assumed that the parties communicate their intentions solely through the haptic channel by applying larger forces. Although this model is valuable for examining the phases of haptic interaction that lead to different role distributions, no experimental study has been conducted to test the model.

The research in human-computer collaboration is more limited. Evrard et al. [3] defined leader and follower roles and realized role exchanges via two distinct functions that allow shifting the control levels using dominance parameters. Even though this system enables a smooth transition between the roles in human-computer collaboration, it lacks a user-centric and dynamic negotiation mechanism. Oguz et al. [11] also utilized leader and follower roles for human-computer collaboration, and equipped the users with a mechanism to negotiate roles dynamically. This framework allowed dynamic and user specific communication with the computer and realized role exchanges systematically using a three-state finite state machine. As proposed in [14], the users were assumed to display their intentions of trading control by dropping or raising the magnitude of the forces they apply through the haptic device.

\section{Virtual TASK}

In this section, we describe the haptic board game application used in the experiments. Also, we explain the haptic negotiation model, as introduced in [11]; and give details on how additional sensory cues are used to communicate the interaction state during the game.

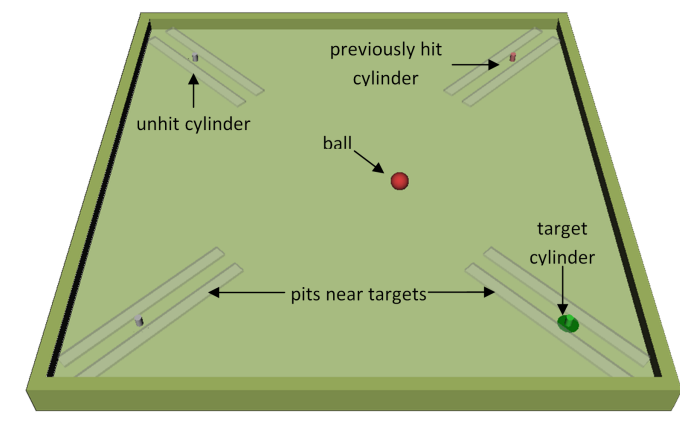

Figure 1: A screenshot of the haptic board game application.

\subsection{Haptic Board Game Application}

A dynamic and interactive haptic board game is used as the virtual environment in the experiments. This application is designed especially to create a dynamic and interactive environment that mimics a physical task, in which the human benefits from the collaboration of a computer. The aim of the game is to hit the cylinders in a specific order in minimum time with fewest faults. In this game, the users are asked to control the position of a ball using a PHANToM Premium haptic device to reach the targets on the board and wait on them to the count of 10 . After this period, a new target is automatically selected. In Figure 1, the previously hit, unhit, and the target cylinders are shown. During the game, the users are instructed to avoid pits that are located at either side of the targets. In case the ball falls into a pit, a fault occurs, all targets that are previously hit are canceled and the user is made to restart the game. The pits are designed to serve as "difficult" regions, where the user is anticipated to demand computer guidance. The task is designed to be relatively "easy" to control outside these regions. Hence, we tried to create an environment in which the human and the computer can perform better than one another at different times. In this regard, this feature makes the game more challenging and makes human-computer collaboration necessary to achieve better task performance. Additionally, as a result of the movement of the ball, the board is tilted in $\mathrm{x}$ and $\mathrm{z}$ axes and the users are fed back with forces that are due to the rotation of the board. Tilting of the board complicates the game for the users so that they are further motivated to demand computer guidance more often.

\subsection{Haptic Negotiation and Role Exchange}

In order to realize role negotiation, the model sketched in Figure 2 is used. In this model, the human and the computer interact with the system using two interface points, namely the Haptic Interface Point (HIP) and the Controller's Interface Point (CIP). These points control the position of a Negotiated Interface Point (NIP), which directly moves the ball.

The $K_{p}$ and $K_{d}$ values shown in the figure denote the stiffness and damping coefficients of the system. By setting $K_{p, C N}$ and $K_{p, H N}$, it is straightforward to set different control levels for the human and the computer. If $K_{p, C N}$ and $K_{p, H N}$ have equal value, the computer and the user will have equal control on movements of the ball. On the other hand, the computer will be the leader if $K_{p, C N}$ has a larger value, and vice versa, the human will be the leader if $K_{p, H N}$ is larger. Hence, this model clearly lets the parties share the control of the ball. Meanwhile it facilitates the process of assigning different control levels (i.e. roles) to the parties.

A smooth transition between different roles is also needed to realize a comfortable negotiation. This dynamic role exchange is realized with the finite state machine shown in Figure 3. The system enters a blending state, which gradually transfers the control level from the user to the computer, or vice versa, during the role ex- 


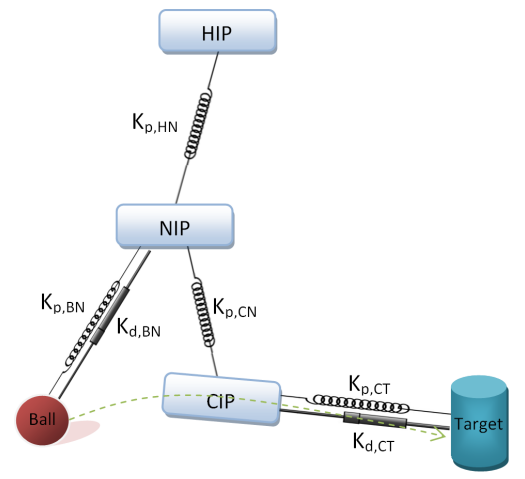

Figure 2: The haptic negotiation model. The roles are changed by setting the stiffness constants, $K_{p, H N}$ and $K_{p, C N}$. These constants are respectively used to set the control levels of the human and the computer.

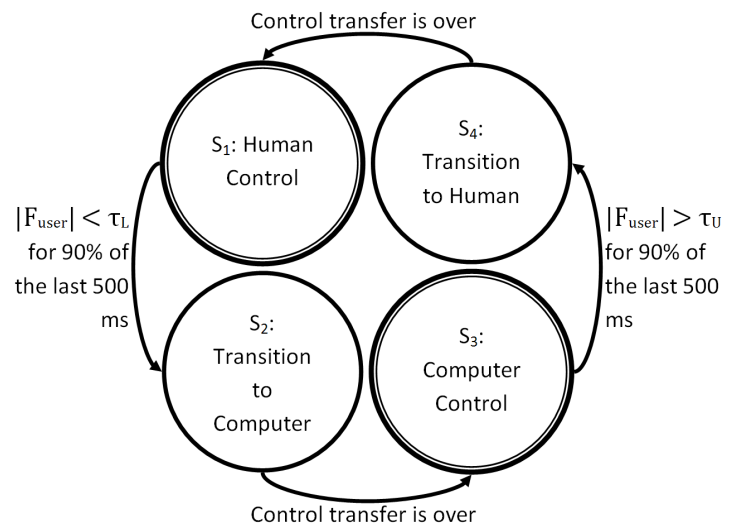

Figure 3: The state diagram defining the role exchange policy. $F_{\text {user }}$ denotes the instantaneous force applied by the user. $\tau_{L}$ and $\tau_{U}$ refer to the personalized lower and upper threshold values for initiating state transitions.

change. We assumed that all intention on realizing role exchanges is displayed by the user through force information. Specifically, we assume that the computer infers the user's intention of getting computer guidance in case the user decreases the forces she applies through the device. Alternatively, we assume that the user wants to stay in control as long as she applies large forces. Initial upper and lower threshold values are set at the beginning of the game. During the course of the game, regarding the user's average forces and the standard deviation of these forces, new upper and lower threshold values are calculated and adaptively updated. The update process is made transparently, and since the range for the thresholds are narrow due to the output capacity of the haptic device, no user reported any inconsistency or difficulty in adapting to the newly calculated thresholds.

\subsection{Additional Sensory Cues}

Haptic negotiation and role exchange allows different means to communicate the system state to the users. We investigate the benefits of utilizing visual and vibrotactile cues to display the interaction state to the users. In this section, we describe how additional sensory cues are displayed within the application.

\subsubsection{Visual Effects}

Two icons, as illustrated in Figure 4(a), are located above the board and grow/shrink to visually illustrate the role exchange process and the control levels of the user and the computer. For instance, if the icon for the user has greater size, the user can understand that she has more control on the movement of the ball.

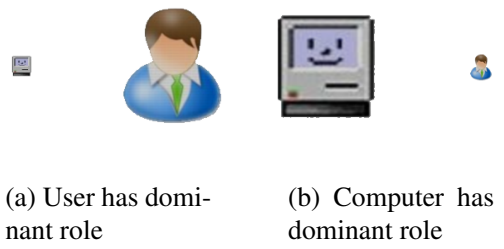

Figure 4: Two different configurations for the role indication icons. In (a) the system is in human control state, whereas in (b) the system is in computer control state.

\subsubsection{Vibrotactile Effects}

In role exchange moments, a high frequency $(100 \mathrm{~Hz})$ vibration is given through the haptic device as a form of buzzing in order to signal that the control is switched from the user to the computer. Also as an allegory to a human's hand tremor that we feel when we work hand to hand with someone, a signal with low frequency at a variable level $(8-12 \mathrm{~Hz})$ is given when the computer has the control of the ball.

\section{EXPERIMENT}

In this section, the experimental conditions, the experiment design, and the measures used in analysis are described.

\subsection{Conditions}

We compared the utility of the role exchange mechanism under the following conditions:

No Guidance (NG) The user plays the game without any computer assistance to control the ball position on the board.

Equal Control (EC) The user and the computer shares control equally at all times to move the ball.

Role Exchange (RE) At any point during the game, the user can hand over/take the control of the ball to/from the computer, by changing the forces she applies through the device.

Visuohaptic Cues (VHC) As in RE condition, the computer infers the user's intentions on taking or giving up control of the game and dynamically changes the degree of control it provides. In addition, role indication icons, buzzing, and tremor will be displayed to the user when appropriate.

\subsection{Procedure and Participants}

30 subjects ( 9 female and 21 male), aged between 21 and 28, participated in our study. Each subject was tested under all four conditions in a day using a within subjects design. Since this study seeks a comparison of the guidance conditions, all subjects were initially presented with NG as a baseline practice condition. Then, they were presented with the guidance conditions (EC, RE, and VHC) in permuted order so that the ordering effects were eliminated. The subjects were instructed in detail about the conditions and the underlying mechanisms. Before starting the experiment, we made sure that the subjects fully understood the role exchange concept and were comfortable in exchanging roles under RE and VHC conditions. In order to avoid any perceptual biases, the guidance conditions were labeled as "Game A", "Game B", and "Game C" in the order the subjects were instructed, whereas NG was labeled 
as the "Practice Game". An experiment consisted of an evaluation and a post-evaluation session. In the evaluation session, the subjects played the game under all conditions for 5 times in each (i.e. 5 trials/condition). Then in the post-evaluation session they played once in each guidance condition and reported the differences in their experience.

\subsection{Measures}

\subsubsection{Quantitative Measures}

For quantitative analysis, we used data acquired in the evaluation session ( 5 trials/condition). We quantify task performance in terms of task completion time. Additionally we examined the efficiency of the user as her work done on the ball per unit of energy she/he spends. In the analysis, the average work done by the user and the average energy spent by her during the task are considered. In order to calculate the average values, the total work and energy are normalized with task completion time.

1. Task performance: The completion time is assumed to represent the performance of the users.

2. Efficiency of the user: The efficiency of the user is a measure of her contribution to the movement of the ball and her effort in doing so. Ideally, we would like the user to highly contribute to the movement of the ball without spending much effort. It should be noted that even though the user may spend much energy during the task, her work done on the ball is mostly affected by her harmony with the computer. In case the user and the computer act against each other, both will spend too much energy but fail to move the ball. Alternatively, if the user often surrenders all control to the computer, even though she spends little energy, she is inefficient since she does almost no work on the ball.

Hence, we introduce an efficiency measure by combining the energy spent by the user and her work done on the ball. The energy spent by the user to complete the task is calculated as the energy spent in moving the haptic interface point by the forces generated through the spring between NIP and HIP:

$$
\text { energy }=\int_{P_{H}}\left|\left(k_{p, H N} x\right) \cdot d x_{H I P}\right|,
$$

where $P_{H}$ is the path traversed by HIP during the trial, $k_{p, H N}$ is the stiffness constant of the spring between HIP and NIP, $x$ is the extension of the spring and $x_{H I P}$ equals to HIP's position.

This work done on the ball by the user can be similarly computed regarding the displacement of the ball and the force acting on the ball due to the user's actions:

$$
\text { work }=\int_{P_{B}}\left|\left(k_{p, H N} x\right) \cdot d x_{\text {ball }}\right|,
$$

where $P_{B}$ is the path traversed by the ball during the trial, $k_{p, H N}$ is the stiffness constant of the spring between HIP and NIP, $x$ is the extension of the spring and $x_{b a l l}$ equals to the ball's position.

Then our efficiency measure is calculated as:

$$
\text { Efficiency }=\frac{\text { work }}{\text { energy }} \times 100,
$$

where work and energy stand for the work done on the ball and the energy spent by the user or the computer during the task. Upon closer inspection, we see that the efficiency is maximized when the user does a large amount of work on the ball with small effort. It should also be noted that the efficiency measure we use is independent of the stiffness and damping coefficients, hence provides means to do a relative comparison between conditions.

\subsubsection{Subjective Measures}

At the end of each experiment, the subjects were given a questionnaire, designed with the technique that Basdogan et al. used previously for investigating haptic collaboration in shared visual environments [1]. The questionnaire asked users to compare their experiences only in the guidance conditions (EC, RE, VHC). Some of the questions were rephrased, and asked again within the questionnaire in random order using a 7-point Likert scale. For evaluation, the averages of the questions, which are rephrased, are considered. Some of the variables investigated in the questionnaire are:

- Collaboration: 2 questions investigated whether the subjects had a sense of collaborating with the computer or not.

- Interaction: 5 questions explored the level of interaction that the subjects experienced during the task.

- Comfort and pleasure: 4 questions investigated how comfortable and pleasurable the task was.

- Trust: 2 questions investigated whether the users believed that their computer partner will aid them in controlling the ball or not.

- Role exchange visibility: A single question explored whether or not the users observed the functionality of the role exchange process during the task.

\section{Results and Discussion}

This section summarizes the quantitative and the subjective evaluation results.

\subsection{Quantitative Measurements}

We measured task completion time and the efficiency of the user during the task as explained in Section 4.3.1. The results are presented in the rest of this section. The means and the standard deviations of the quantitative metrics for each guidance condition can be seen in Table 1. Any statistical difference between conditions is investigated using t-test.

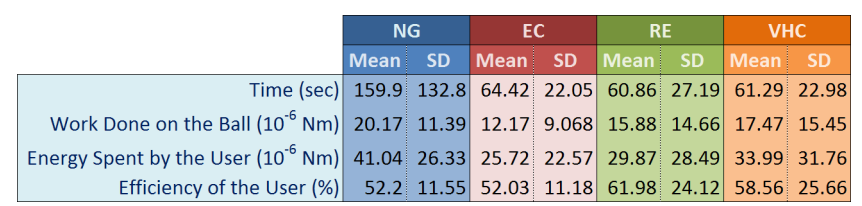

Table 1: Means and standard error of the means of the guidance conditions for the objective measurements

\subsubsection{Task Performance}

The average completion times (in seconds) under each condition is plotted in Figure 5. The inset in the upper right corner of the figure shows a close-up of the means of the guidance conditions (EC, RE, VHC). As seen in the figure, all guidance conditions improve completion time when compared to NG. T-test indicates a statistically significant difference between NG and all three guidance conditions in terms of completion time ( $p$-value $<0.01)$. Also, the average completion time under EC condition is observed to be higher than those in RE and VHC conditions. As a result, we conclude that the role exchange schemes (RE and VHC) improve completion time more than an equal control guidance scheme (EC) does. We also observe that although adding certain sensory cues seem to increase the completion time (VHC), this increase is not statistically significant $(p$-value $<0.05)$. 


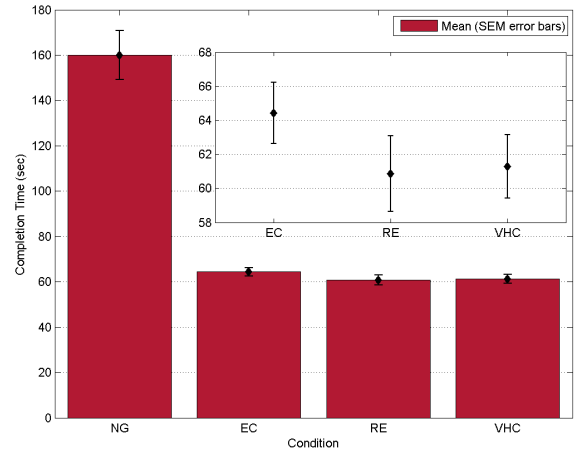

Figure 5: Comparison of the task completion time under the different conditions. Since NG displays high variance, the inset figure provides a closeup of the comparison of the guidance conditions only.

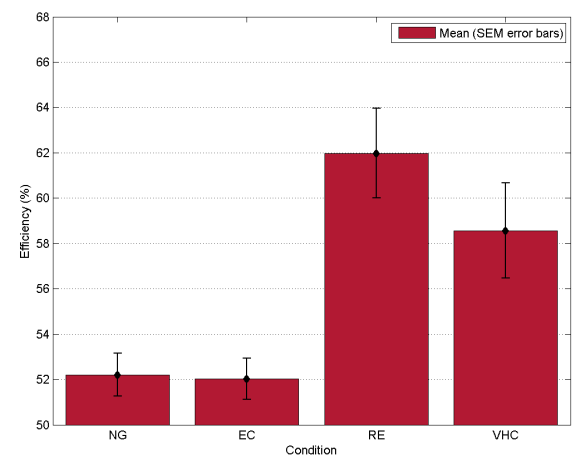

Figure 6: Comparison of the efficiency of user under the different conditions

\subsubsection{Efficiency of the User}

Figure 6 shows a comparison of the means of the efficiencies of the users under each guidance condition. The efficiencies of the users under NG and EC are significantly lower than those of the role exchange conditions (RE and VHC) $(p$-value $<0.01)$. The highest efficiency is observed under RE. T-test indicates that the differences between NG and EC and between RE and VHC are not statistically significant ( $p$-value $<0.05$ ). As a result, we conclude that the the users work more efficiently under a role exchange scheme then they do under an equal control scheme.

Figures 7 and 8 respectively illustrate the average energy spent by the users and the average work done on the ball under each condition. The users have to control the ball without any assistance in NG, hence they spend the largest energy under this condition. Also, the work done on the ball by the users is maximized in NG. T-test revealed that the average energy consumption of the users and their work done on the ball under NG is significantly larger than those in three guidance conditions ( $p$-value $<0.05$ ).

The guidance conditions, in ascending order of the amount of energy that the users spend, can be listed as EC, RE, and VHC. However, no statistically significant difference is observed between these conditions ( $p$-value $<0.01)$. The work done on the ball under EC is smaller than that under RE and VHC ( $p$-value $<0.05)$. Hence, we conclude that the users tend to surrender to computer control in EC, and even though they spend a small energy, they fail in controlling the ball. On the other hand, in NG, since controlling the ball without guidance is hard, even though the users can

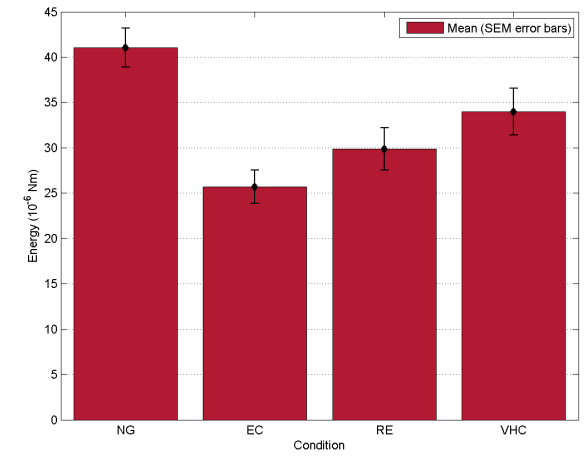

Figure 7: Comparison of the average energy spent by the user under different conditions

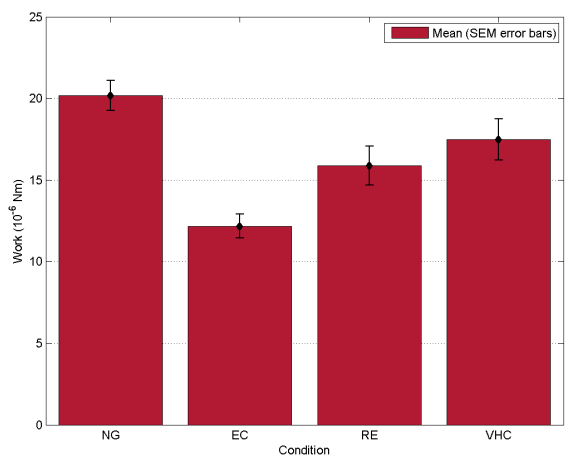

Figure 8: Comparison of the average work done on the ball by user under different conditions

move the ball, they spend a large amount of energy in doing so. As a result, we observe that the users work most efficiently when role exchange is present. However, adding different sensory cues decrease the efficiency slightly, probably due to the extra cognitive load they introduce to the task.

\subsection{Subjective Measures}

The subjective evaluation was done only for the guidance conditions, hence this section illustrates a comparison only between RE, $\mathrm{EC}$, and VHC conditions. Table 2 lists the means and standard deviations of subjects' questionnaire responses regarding the evaluated variables. It also demonstrates the pair-wise comparisons of the guidance conditions for the subjective measures, as summarized in Section 4.3.2, obtained by Wilcoxon Signed-Rank Test using $p$ value $<0.05$. For each pair of guidance conditions, the one with the higher mean is marked in the table. Also the condition pair is check-marked in case the conditions bear significant differences in terms of the variable in the corresponding row.

The subjective evaluation results can be summarized as follows:

- Collaboration: The sense of collaboration during the task is significantly higher when additional sensory cues are displayed to the user to indicate the control state.

- Interaction: The interaction level of the task is significantly higher when additional sensory cues are displayed.

- Comfort and pleasure: When compared to an equal control condition, the users find the interface significantly more com- 


\begin{tabular}{|r|rr|r|r|r|r|r|r|r|r|}
\multicolumn{1}{c}{} & \multicolumn{2}{c}{ EC } & \multicolumn{2}{c|}{ RE } & \multicolumn{2}{c|}{ VHC } & & \\
& Mean & SD & Mean & SD & Mean & SD & EC - RE & EC - VHC & RE - VHC \\
\hline Collaboration & 4.2 & 1.35 & 4.47 & 1.14 & 5.01 & 0.86 & $\mathbf{x}$ & $\checkmark$ & $\checkmark$ \\
Interaction & 4.12 & 1.17 & 4.47 & 0.87 & 4.84 & 0.67 & $\mathbf{x}$ & $\checkmark$ & $\checkmark$ \\
Comfort and pleasure & 4.12 & 1.04 & 4.32 & 0.91 & 4.62 & 0.93 & $\mathbf{x}$ & $\checkmark$ & $\mathbf{x}$ \\
Trust & 4.18 & 1.57 & 4.57 & 1.47 & 5.2 & 0.92 & $\mathbf{x}$ & $\checkmark$ & $\checkmark$ \\
Role exchange visibility & 3.27 & 1.99 & 3.87 & 2.03 & 4.67 & 1.56 & $\mathbf{x}$ & $\checkmark$ & $\checkmark$ \\
\hline
\end{tabular}

Table 2: Pair-wise comparisons of the guidance conditions for the subjective measurements. The differences that are statistically significant according to the Wilcoxon Signed-Rank Test for $p$-value $<0.05$ are marked.

fortable, enjoyable, and easier to perform, only if additional sensory cues are provided to them.

- Trust: A role exchange scheme lets the users trust in the computer's control during collaboration, such that they believe that it will move the ball correctly. This sense of trust is significantly higher when additional sensory cues are present.

- Role exchange visibility: Additional sensory cues make the role exchange process significantly more visible so that the users can track the current state of the system more successfully when these cues are present.

\section{Conclusions}

This paper presents the key results of an experimental study on the utility of a role exchange mechanism that enables humancomputer collaboration as a shared control scheme. We defined leader and follower roles for the user and the computer. Using the role exchange mechanism, the human negotiates with the computer through force information to change her control level on the task. Even though negitiating through force information lets the users interact dynamically and smoothly with the computer, it should be noted that this scheme may invoke accidental role exchanges in case the user decreases the forces she applies as an attempt to do fine-positioning. In order to overcome such problems, a sophisticated scheme which can infer the user's motivation is required. As a future work, we will explore the utility of statistical learning models to discover certain human characteristics regarding gameplay to better infer the user's intentions. We hypothesize that these characteristics will be useful to program the computer differently to achieve a more personal and effective interaction scheme.

We observed that the role exchange mechanism improves task performance and maximizes the efficiency of the user when compared to an equal control guidance condition and a condition in which no guidance exists. In comparison to our earlier work [11], this result shows that the users can benefit from a role exchange mechanism when they are explicitly instructed on the principles of negotiating with the computer. We also investigated the benefits of adding visual and vibrotactile cues on top of the role exchange mechanism to directly inform the users on their and the computer's control levels at a given time during the task. We observed that the additional cues slightly decreased the users' efficiencies, but improved the sense of collaboration and the level of interaction during the task. The users additionally reported that they found the task more comfortable and easier to perform in the existence of these cues, and stated that it was significantly easier to understand the interaction state and their control levels on the task. Finally we observed that the users' trust towards computer's guidance capability was significantly higher when informative cues were displayed to them. However, we should note that no usability studies has been conducted in designing how these additional cues should be displayed to the user. Hence we presume that the combination of visual and vibrotactile cues can be inconsistent and may result with an increase in the user's cognitive load. A careful evaluation is required on how to design such cues, depending on the nature and the requirements of the task.

As a result, we suggest that a role exchange mechanism could be beneficial especially in training applications. With such a role exchange scheme, the users of the system can take initiative to choose whether they will use the computer guidance or not. We also emphasize that although equipping the role exchange mechanism with additional cues can improve the task subjectively, it can also deteriorate the performance and the efficiency of the users.

\section{REFERENCES}

[1] C. Basdogan, C.-H. Ho, M. A. Srinivasan, and M. Slater. An experimental study on the role of touch in shared virtual environments. ACM Trans. Comput.-Hum. Interact., 7(4):443-460, 2000.

[2] P. Dillenbourg, M. Baker, A. Blaye, and C. O'Malley. The evolution of research on collaborative learning. In E. Spada \& P. Reiman (Eds) Learning in Humans and Machine: Towards an interdisciplinary learning science, pages 189-211. Elsevier, Oxford, 1996.

[3] P. Evrard and A. Kheddar. Homotopy switching model for dyad haptic interaction in physical collaborative tasks. In WHC '09: Proceedings of the World Haptics 2009 - Third Joint EuroHaptics conference and Symposium on Haptic Interfaces for Virtual Environment and Teleoperator Systems, pages 45-50, Washington, DC, USA, 2009. IEEE Computer Society.

[4] D. Feygin, M. Keehner, and F. Tendick. Haptic guidance: Experimental evaluation of a haptic training method for a perceptual motor skill. In HAPTICS '02: Proceedings of the 10th Symposium on Haptic Interfaces for Virtual Environment and Teleoperator Systems, page 40, Washington, DC, USA, 2002. IEEE Computer Society.

[5] B. A. C. Forsyth and K. E. MacLean. Predictive haptic guidance: Intelligent user assistance for the control of dynamic tasks. IEEE Transactions on Visualization and Computer Graphics, 12(1):103$113,2006$.

[6] J. Huegel and M. O’Malley. Progressive haptic and visual guidance for training in a virtual dynamic task. In Haptics Symposium, 2010 IEEE, pages $343-350$, mar. 2010.

[7] J. Lee and S. Choi. Effects of haptic guidance and disturbance on motor learning: Potential advantage of haptic disturbance. In Haptics Symposium, 2010 IEEE, pages 335 -342, march 2010.

[8] J. Moll and E.-L. Sallnäs. Communicative functions of haptic feedback. In Proceedings of the 4th International Conference on Haptic and Audio Interaction Design, HAID '09, pages 1-10, Berlin, Heidelberg, 2009. Springer-Verlag.

[9] D. Morris, H. Tan, F. Barbagli, T. Chang, and K. Salisbury. Haptic feedback enhances force skill learning. In WHC '07: Proceedings of the Second Joint EuroHaptics Conference and Symposium on Haptic Interfaces for Virtual Environment and Teleoperator Systems, pages 21-26, Washington, DC, USA, 2007. IEEE Computer Society.

[10] I. Oakley, S. Brewster, and P. Gray. Can you feel the force? an investigation of haptic collaboration in shared editors. In In Proceedings of Eurohaptics, pages 54-59, 2001.

[11] S. Oguz, A. Kucukyilmaz, T. Sezgin, and C. Basdogan. Haptic negotiation and role exchange for collaboration in virtual environments. In Haptics Symposium, 2010 IEEE, pages 371 -378, mar. 2010.

[12] B. Plimmer, A. Crossan, S. A. Brewster, and R. Blagojevic. Multimodal collaborative handwriting training for visually-impaired people. In Proceeding of the twenty-sixth annual SIGCHI conference on Human factors in computing systems, CHI '08, pages 393-402, New York, NY, USA, 2008. ACM.

[13] K. B. Reed and M. A. Peshkin. Physical collaboration of humanhuman and human-robot teams. IEEE Trans. Haptics, 1(2):108-120, 2008.

[14] N. Stefanov, A. Peer, and M. Buss. Role determination in humanhuman interaction. In WHC '09: Proceedings of the World Haptics 2009 - Third Joint EuroHaptics conference and Symposium on Haptic Interfaces for Virtual Environment and Teleoperator Systems, pages 51-56, Washington, DC, USA, 2009. IEEE Computer Society. 